\title{
Comparación sistemática de metodologías basadas en información mutua para el registro multimodal de imágenes médicas
}

\author{
Systematic comparison of mutual information-based methodologies for the \\ multimodal register of medical images
}

\author{
Gustavo Adolfo Ospina Torres, Walter Serna Serna, Genaro Daza Santacoloma \\ Instituto de Epilepsia y Parkinson del eje cafetero, Pereira, Colombia \\ researcheneurocentro.com.co \\ gaotcom2eutp. edu.co
}

\begin{abstract}
Resumen-El registro de imágenes sobre todo en el ámbito de la medicina, han cobrado una gran importancia en las últimas décadas pues esto se mejora la planeación, intervención y posterior análisis de dichos procedimientos médicos, como es el caso de las cirugías guiadas por imágenes donde se requiere una especial precisión y confiabilidad. Registrar dos o más imágenes consiste en alinearlas de tal manera que queden orientadas de la misma forma, así se puede combinar la información de ambas en un mismo espacio y en una misma representación visual.
\end{abstract}

El objetivo principal de este trabajo es comparar de forma sistemática algunas metodologías para el registro de imágenes multimodales utilizando medidas de similitud basadas en información mutua (IM).

Se obtuvo que el registro de imágenes basada en IM es capaz de registrar imágenes multimodales y monomodales con poca susceptibilidad a los cambios de brillo o contraste en la imagen, pero con alta sensibilidad al ruido. También se estableció que las técnicas de optimización basadas en enjambres de partículas tienen un mejor desempeño para el resultado final de registro en comparación con las otras técnicas analizadas. Además, la técnica de interpolación cúbica (transformación espacial) mejora la confiabilidad del algoritmo en imágenes multimodales.

Palabras clave- Información mutua, optimización, procesamiento de imágenes, registro de imágenes

Abstract - the image registration in medical application have become very important topic in recent decades, because the planning, intervention and subsequent analysis of these medical procedures are improved, such as of imaging guided surgery where is required an especial precision and reliability. Image registration consists in align the images so that they are oriented in the same way, so you can combine information from both images in the same space and the same visual representation

The main objective of this work is to compare systematically some methodologies for multimodal image registration using measures based in mutual information (MI).

We found that the image registration based MI is able to register multimodal and monomodal images with little susceptibility to changes in brightness or contrast in the image, but with high sensitivity to noise. We also established that optimization techniques based on particle swarms have better performance for the registration compared to other techniques analyzed. Furthermore, the technique of cubic interpolation (spatial transformation) improve the reliability of the algorithm in multimodal images.

Key Word - Image Processing, Image Registration, Mutual Information, Optimization.

\section{INTRODUCCIÓN}

El registro de imágenes consiste en alinear espacialmente dos o más imágenes, combinando su información en una sola representación visual. Por ejemplo, alinear un conjunto de imágenes de resonancia magnética con imágenes de tomografía computarizada, las primeras permiten explorar los tejidos blandos del paciente, mientras que las segundas resaltan los tejidos rígidos, la mezcla de ambas brinda la información adecuada para procedimientos diagnósticos y tratamientos quirúrgicos. En tal sentido, el registro de imágenes permite la visualización simultánea de ambos tipos de imágenes, alineadas, escaladas y fusionadas.

El registro de las imágenes consiste en las siguientes operaciones: 1) Transformación espacial: modifica geométricamente una de las imágenes con el fin de alinearla 
con respecto a otra, 2) Comparación: función que permite establecer la similitud entre un conjunto de imágenes transformadas y 3) Optimización: realiza la búsqueda de los mejores parámetros de transformación espacial con respecto a los resultados de la función o medida de comparación.

Una gran dificultad en el proceso de registro es el hecho de que las imágenes pueden ser adquiridas por sensores diferentes como tomografía, resonancia magnética, SPECT, PET, angiografía, ultrasonido, entre otras, por lo cual la representación de los valores de los pixeles en la escena puede ser diferente a pesar de estar analizando una misma estructura corporal. Estos conjuntos de imágenes se conocen como imágenes multimodales. Cuando las imágenes son provenientes del mismo sensor, pero tomadas en lapsos de tiempo diferentes, o en orientaciones espaciales distintas, se llaman imágenes monomodal.

Como medida de comparación, la información mutua (IM) es una de las más ampliamente utilizadas en los procesos de registro de imágenes multimodales [1], sin embargo, existe una gran cantidad de variaciones para las distintas partes del proceso de registro basado en IM, como, por ejemplo: el uso de distintos tipos de técnicas de optimización, transformación espacial (interpolación) y mejoras sobre la medida de comparación por IM. Por tanto, es difícil establecer cuáles métodos son los que presentan mejor desempeño para el registro basado en IM.

El objetivo principal de este trabajo es comparar de forma sistemática algunas metodologías para el registro de imágenes multimodales utilizando medidas de similitud basadas en IM.

Se analizaron diferentes metodologías para el registro de imágenes multimodales con base en IM, empleando diferentes técnicas para la transformación espacial, la optimización y considerando mejoras sobre la función de costos (medida de comparación), además se evaluó el comportamiento de cada configuración en situaciones de variabilidad de las imágenes médicas, es decir, en condiciones de ruido, correcciones gamma y ecualizaciones del histograma. En tales casos se obtuvo que el registro de imágenes basada en IM es capaz de registrar imágenes multimodales y monomodales con poca susceptibilidad a los cambios de brillo o contraste en la imagen, pero con alta sensibilidad al ruido. También se estableció que las técnicas de optimización basadas en enjambres de partículas tienen un mejor desempeño para el resultado final de registro en comparación con las otras técnicas analizadas. Además, la técnica de interpolación cúbica (transformación espacial) mejora la confiabilidad del algoritmo en imágenes multimodales.

\section{MARCO TEÓRICO}

El registro de imágenes médicas permite combinar la información de diferentes fuentes de información y visualizarlas en una única imagen con el objetivo de facilitar la interpretación durante la examinación y/o tratamiento clínico [2].

Según [3], las metodologías de registro de imágenes se pueden dividir en dos tipos: 1) Metodologías basadas en características, en las cuales se extraen vía segmentación de la imagen algún tipo de característica morfológica como puntos, líneas, bordes, etc., que luego son comparadas; sin embargo se debe tener en cuenta que son semiatomáticas, es decir, requieren de un usuario entrenado para que señale los puntos morfológicos de interés, tal dependencia puede generar errores de segmentación y por ende errores en el registro, sin embargo el hecho de que las características sean simples de calcular reduce los tiempos de cómputo autónomos. 2) Metodologías basadas en intensidad, en las que se comparan los valores de intensidad de los vóxeles y píxeles por medio de una medida que determina que tan semejantes son las imágenes entre sí, este tipo de metodologías son típicamente demandantes computacionalmente, además cambios abruptos en los valores de intensidad de los vóxeles (pixeles) pueden alterar radicalmente los resultados, este tipo de cambios se dan en condiciones de saturación de captura, por presencia de ruido y artefactos en la escena. Una ventaja de estas metodologías es que no requieren de etapa de segmentación previa y se pueden realizar de manera totalmente automática.

Hay varios factores esenciales para el proceso de registro, y estos son: la imagen de referencia o fija, la cual permanece sin cambios durante el registro, la imagen móvil u objetivo la cual cambia para adecuarse a la imagen de referencia, y la transformación espacial o geométrica que es necesaria hacer para registrar la imagen objetivo a la imagen de referencia. Para que exista correspondencia entre la imagen de referencia y la imagen objetivo es necesario que una medida de comparación, usualmente de tipo estadística, llamada medida de similitud, refleje un alto valor al comparar la imagen con relación a un conjunto de parámetros espaciales que alineen las imágenes, por lo tanto, es necesario el uso de una metodología de optimización que maximice la medida similitud (o minimice una medida de disimilitud). Con posterioridad a esto, es necesario el uso de un sistema de interpolación con el fin de aproximar los valores de los píxeles que han sido transformados a posiciones no rígidas y por último se procede con la fusión de las imágenes por medio de un promedio de los valores de intensidad de las dos imágenes.

En este trabajo en particular se comparan de forma sistemática algunas técnicas de registro basadas en intensidad de vóxeles e información mutua, dichas técnicas dominan el campo del registro de imágenes médicas multimodales [4].

\footnotetext{
A. Modelos de transformaciones geométricas o espaciales
} 
Las transformaciones geométricas juegan un papel central en el proceso de registro. Estos modelos imponen una distorsión geométrica que se aplica sobre las imágenes [5]. Existen dos tipos de transformaciones, rígidas y no rígidas.

\section{Transformaciones rígidas}

Debido a su naturaleza lineal preservan todas las distancias y ángulos internos de las imágenes, esta transformación incluye rotación desde el centro de la imagen, y traslación, el proceso se representa por:

$$
T_{\mu}\left(x^{\prime}\right)=R\left(x^{\prime}-c\right)+t+c
$$

Donde $x^{\prime}$ es un vector con la coordenada $(x, y)$ de la imagen, $\mu=\left(\theta, t_{x}, t_{y}\right)^{T}$, representa los parámetros de translación $t$ en la coordenada $(x, y)$ de la imagen y rotación $\theta$, asi $R$ es la matriz de rotación, $3 x 3$ para el caso de dos dimensiones y $4 \times 4$ en el caso de 3 dimensiones y $t$ es un vector de traslación para $(x, y)$ y $c$ es escalar con el centro de la rotación [2].

Dentro de las transformaciones rígidas se encuentra la transformación por similitud, en la cual la imagen puede trasladarse, rotarse y escalarse, tal que,

$$
T_{\mu}\left(x^{\prime}\right)=\mathrm{s} R\left(x^{\prime}-c\right)+t+c
$$

Donde $s$ es un escalar que indica la escala isotrópica de la imagen.

\section{Transformaciones no rígidas}

Estas transformaciones pueden deformar libremente la imagen original, en algunos casos generan una malla deformable en la imagen, así esta malla puede cambiar en determinadas partes de la imagen, es muy útil cuando se tienen estructuras blandas en el cuerpo humano que se deformarán al realizar alguna intervención sobre ellos. Una transformación no rígida muy común es basada en B-spline representada por:

$-n$

$$
T \mu(x)=x^{\prime}+\sum p_{k} \beta^{3}\left(\frac{x-x_{k}}{\sigma}\right)
$$

Donde $X_{k}$ son los puntos de control, $\beta^{3}$ es un polinomio Bspline multidimensional cúbico, $p_{k}$ son los coeficientes de la B-spline, $\sigma$ son los espacios de los puntos de control. El objetivo de esta transformación es deformar o mover la malla formada por los puntos de control en la imagen objetivo [2].

\section{Interpolación}

Estas técnicas pueden usarse tanto en las transformaciones rígidas como en las no rígidas, y es necesaria cuando los puntos en una imagen son mapeados a posiciones no fijas (posiciones en puntos intermedios la cuadricula determinada por los píxeles de la imagen) después de la transformación [6]. Algunas técnicas comunes de interpolación son:

- Interpolación lineal: La interpolación Lineal utiliza dos puntos conocidos para obtener un tercero interpolado de los dos.

- Vecinos más cercanos: utiliza el valor de los vecinos más cercanos al vóxel o píxel analizado para obtener un valor aproximado del punto interpolado.

- Interpolación bilineal: usa una interpolación lineal pero aplicada a dos variables, utiliza 4 vecinos más cercanos para calcular el valor promedio del vóxel a representar.

- Convolución cúbica: es semejante a la interpolación bilineal, pero utiliza 16 vecinos al vóxel bajo análisis.

B. Algoritmos para la comparación de imágenes

Estos algoritmos evalúan características presentes en las imágenes, con el fin de establecer un nivel de correspondencia. Existen varios tipos de medidas de similitud tales como: suma del cuadrado de las diferencias, coeficiente de Pearson, radio mínimo, que son ineficaces para la comparación de imágenes multimodales, pues generalmente se basan en la comparación directa de las intensidades de las imágenes [4], los cual es inadecuado porque en el caso de imágenes multimodales (provenientes de fuentes de registro diferentes), los valores de intensidad entre dos imágenes pueden ser muy diferentes, en estos casos las medidas mencionadas pueden reportar que las imágenes en registro no son correspondientes entre ellas, lo cual es incorrecto. Por lo tanto, es necesario el uso de una medida de comparación que no sea tan sensible a la información puntual de la intensidad por vóxel (píxel), en tal sentido, las técnicas basadas en información y/o entropías son de gran ayuda.

\section{Información mutua}

Esta medida de similitud consiste en minimizar la entropía conjunta de las imágenes y por tanto maximizar su información mutua [1] [4]. La información mutua (IM) se define como:

$$
\mathrm{IM}(\mathrm{A}, \mathrm{B})=\mathrm{H}(\mathrm{A})+\mathrm{H}(\mathrm{B})-\mathrm{H}(\mathrm{A}, \mathrm{B})
$$

Donde H(A) y H(B), es la entropía de Shannom (en honor a Claude E. Shannon) de la imagen A y B respectivamente y $\mathrm{H}(\mathrm{A}, \mathrm{B})$ es la entropía conjunta de las imágenes definidas en la ecuación 5,6 y 7, respectivamente.

$$
\begin{gathered}
\mathrm{H}(A)=-\sum_{a \in A} p_{A}(a) \log p_{A}(a) \\
H(B)=-\sum_{b \in B} p_{B}(b) \log p_{B}(b) \\
H(A, B)=-\sum_{a \in A} \sum_{b \in B} p_{A B}(a, b) \log p_{A B}(a, b)
\end{gathered}
$$


Donde $P_{A}(a)$ y $P_{B}(b)$ es la probabilidad de encontrar un vóxel o píxel en la imagen $A$ o $B$ y $P_{A B}(a, b)$ es la probabilidad conjunta, para este caso es necesario el histograma conjunto dividido por el número total de píxeles en la imagen [4]. El histograma conjunto es una herramienta usada para la visualización de la relación entre correspondencia de intensidades de una o más imágenes.

La información mutua de Shannon se usa para medir la dispersión de la densidad de probabilidades conjuntas, cuando ésta dispersión es mínima la dependencia de las intensidades de los píxeles (vóxeles) es máxima [1].

\section{Información mutua normalizada}

Una mejora a la IM está basada en el trabajo de Studholme [7], conocida como información mutua normalizada IMN, según [1], es una versión más robusta que la IM estándar. La IM normalizada está definida como.

$$
\operatorname{IMN}(A, B)=\frac{H(A)+H(B)}{H(A, B)}
$$

La IMN tiene un mejor desempeño ante el ruido y condiciones cambiantes en la imagen, como brillo y contraste.

\section{Algoritmos de Optimización matemática}

Cada algoritmo de registro requiere un algoritmo de optimización, el cual busca la óptima transformación espacial para minimizar o maximizar una función de coste o medidas de similitud, ver [6]. Un algoritmo de optimización trata buscar cuál es el valor donde es mínimo cierta función ya sea en una o en varias dimensiones o con ciertas condiciones (restricciones). Para un algoritmo de optimización dado se puede expresar como.

$$
\mathrm{T}_{\text {optimo }}=\operatorname{argmin}_{\mathrm{T}}\left(\mathrm{f}\left(\mathrm{T}\left(\mathrm{X}_{\mathrm{s}}, \mathrm{X}_{\mathrm{R}}\right)\right)\right.
$$

Donde $\mathrm{T}$ es la transformación y f es la función de coste a ser minimizada o maximizada. Existen varios métodos y se pueden dividir en optimizadores globales o locales. Los locales solo pueden encontrar valores mínimos localizados dado ciertos valores iniciales. La búsqueda no siempre hallará el valor mínimo de toda la función. Los optimizadores globales tratarán de buscar siempre el mínimo global en toda la función.

Algunos algoritmos de optimización están basados en comportamientos biológicos, como por ejemplo el proceso de alimentación o de cría de ciertas especies de animales. Estos han inspirado métodos de búsqueda para la solución de problemas de optimización [8]. Estos algoritmos reciben el nombre de algoritmos evolutivos. Algunos de ellos son:

- Evolución diferencial (diferential evolution DE): es un tipo de algoritmo evolutivo para búsquedas sobre espacios continuos desarrollado por Storn y Price [8]. En este método una población inicial es generada aleatoriamente. En cada iteración se generan una nueva población evolucionada de la anterior. Si la nueva población es mejor que la anterior, la nueva población la reemplazará, si no es descartada.

- Búsqueda Cuco (Cuckoo search CS): este método de optimización metaheurístico está basado en el comportamiento reproductivo de algunas especies de pájaros conocidas como cucos. Desarrollado por Xin She Yang y Suash Deb en [9].

- Algoritmo de optimización por enjambre de partículas (Basic Particle Swarm optimization PSO): es un método de optimización heurístico propuesto por Eberhart y Kennedy como se aprecia en [10], está inspirado en el comportamiento social de agentes encontrados en la naturaleza. Éste comportamiento puede observarse en pájaros sociales, enjambres de abejas, escuelas de peces entre otros.

D. Técnicas para la evaluación del registro.

Desde el punto de vista del usuario la precisión y exactitud son más importante en sí que el método de registro obtenido, sobretodo en medicina donde el conocimiento absoluto de la precisión es necesario para tomar decisiones apropiadas. Cinco criterios se pueden evaluar para registro de imágenes médicas según [11].

- Precisión: se refiere a la diferencia en distancias entre la imagen objetivo y la de referencia. Generalmente se escogen imágenes en las cuales las distancias son conocidas luego se obtiene el error en la distancia entre puntos conocidos, ese error puede ser medio, máximo o medio cuadrático RMS.

- Confiabilidad: trata del número de veces que el algoritmo encuentra una respuesta satisfactoria comparada con el número total de pruebas realizadas. Si se hacen $n$ pruebas a $n$ pares de imágenes y $m$ de n son el número de veces que el algoritmo encuentra una respuesta satisfactoria, si $n$ es lo suficientemente grande entonces $m / n$ representara la confiabilidad del algoritmo. Entre más cerca esté el radio a 1 más confiable será el algoritmo.

- Robustez: es el grado de estabilidad del algoritmo bajo condiciones cambiantes de los parámetros de entrada. Puede ser evaluado con respecto al ruido, intensidad, diferencias geométricas, oclusión, etc. Por lo general si un algoritmo es poco preciso y no confiable también se dice que es poco robusto.

- Complejidad computacional: la cual determina la rapidez del algoritmo y muestra cuán práctico es el 
algoritmo en situaciones reales. La complejidad computacional es la medida del número de adiciones/multiplicaciones expresadas como función de las dimensiones de la imagen.

Evaluar estos parámetros dentro de un método de registro en lo general no es una tarea fácil según [11], pues no existe un estándar general con lo cual se pueda analizarlas, por lo tanto, es difícil establecer comparaciones entre ellas pues resultan incompatibles metodológicamente.

\section{MARCO EXPERIMENTAL}

Como se expresó anteriormente, el registro de imágenes consta de una transformación espacial, con su interpolador, una medida de comparación, y una etapa de optimización matemática. Cada uno de estos pasos posee cierto número de técnicas y parámetros que afectan el proceso de registro. El objetivo fue establecer algunos de estos parámetros y técnicas para el registro multimodal y monomodal de imágenes médicas, con el fin de una futura implementación en sistemas de navegación quirúrgica.

Los algoritmos de registro fueron diseñados inicialmente en el entorno Matlab, pero posteriormente fueron implementados en el lenguaje C\#, para esto se recurrió a dos librerías computacionales, las cuales fueron: OpenCV con su wrapper para C\# OpenCVSharp, encargada del procesamiento de imágenes; y la librería LibOptimizaction, encargada de los algoritmos de optimización matemática.

En el diseño del algoritmo se realizaron varias pruebas para cuantificar el desempeño de las diferentes partes y así escoger una combinación de parámetros adecuados. El computador utilizado en las pruebas fue un AMD Athlon II P320 Dual core a $2.10 \mathrm{GHz}$, con $3 \mathrm{~Gb}$ de RAM a $1333 \mathrm{MHz}$. Sin embargo, también se realizaron algunas pruebas en un computador Intel Core Vpro I7-4940MX 3.10GHz con 4 procesadores físicos y 8 hilos, con $32 \mathrm{~Gb}$ de RAM a $1600 \mathrm{MHz}$. Se debe recalcar que no se utilizaron todos los núcleos de este procesador.

Entre las técnicas y parámetros analizados se tienen:

- En las pruebas se utilizaron tanto transformaciones espaciales rígidas como de similitud, sin embargo, se compararán los métodos de interpolación: Cúbico, lineal, vecinos más cercanos y Lanczos4, en búsqueda del más confiable, también se analizó el tiempo de procesamiento de estos.

- En la etapa de comparación de imágenes, aunque se optó por el uso de la información mutua (IM), se examinó la diferencia entre la información mutua normalizada (IMN) y no normalizada, según [1] la IMN es más robusta que la IM. Para esto se plantearon pruebas de robustez entre las dos técnicas, como el cambio de estas ante ruido gaussiano, cambios ante filtros eliminadores de ruido (mediano y Weiner) y comportamientos ante correcciones gamma y ecualizaciones del histograma (estándar y CLAHE) en las imágenes.

- En la etapa de optimización matemática, se compararon algunas técnicas de optimización, entre las cuales se tienen: evolución diferencial, búsqueda Cuco y optimizadores por enjambre de partículas con 3 variantes: peso inercial adaptativo, peso inercial caótico y decrecimiento lineal. Algunos parámetros libres de las técnicas fueron analizados en búsqueda del menor tiempo de ejecución, pero se comparó principalmente su confiabilidad.

- El algoritmo de registro diseñado está planeado para poder registrar imágenes multimodales, por lo tanto, se realizó una prueba de precisión del algoritmo con base en imágenes de TAC y angiografía, de un phantom, ver Imagen 1. Dado que las imágenes de TAC y angiografía, son muy diferentes entre sí, es de esperar no obtener un registro exacto de las dos imágenes.

El algoritmo fue probado en los siguientes 4 tipos de imágenes:

- Set1: conjunto de dos imágenes multimodales (angiografía y TAC) provenientes de un phantom, como se ven en la imagen 1. Como las imágenes de TAC son cortes y las imágenes de angiografía son proyecciones al plano similar a los rayos $\mathrm{X}$, los cortes de TAC se han sumado para recrear una imagen similar a la de angiografía, ver imágenes 1,2.

- Set2: conjunto de imágenes multimodales de una rodilla tomadas con dos tipos de secuencia de MRI. Ver imagen 3

- Set3: el mismo conjunto que en el set2, pero con previo escalamiento e inclinación, solo varían en el ángulo y translación.

- Set4: conjunto de imágenes monomodales (solo TAC) del phantom de la imagen 1, esta imagen fue usada como control pues se conocía la diferencia en el ángulo y la translación entre las dos imágenes. Ver imagen 4 . 


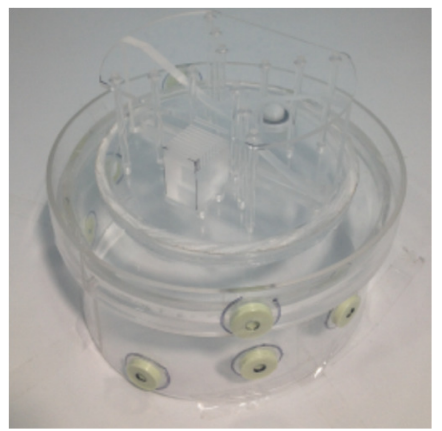

Imagen 1: Imagen de un Phantom de acrílico utilizado por el grupo Applied Neuroscience, el cual posee estudios en MRI, TAC y angiografía.
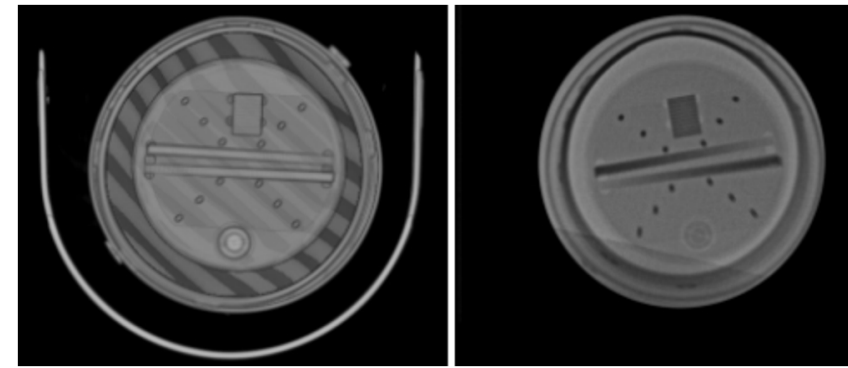

Imagen 2: izquierda proyección de cortes de TAC del Phantom, derecha: estudios de angiografía del Phantom de la imagen 1

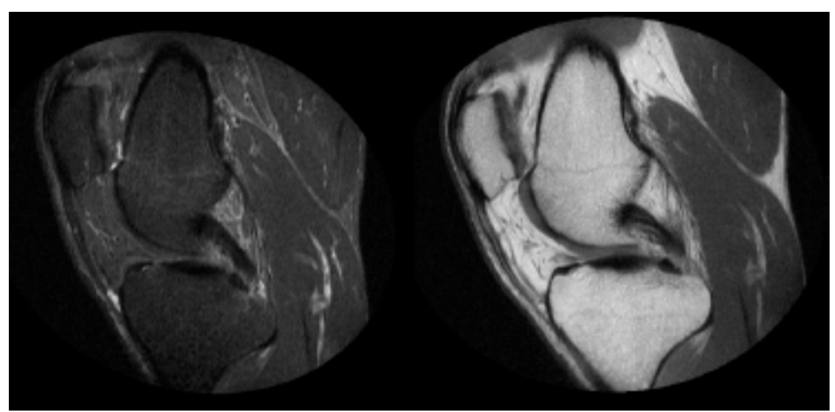

Imagen 3: Imagen de rodilla en MRI con dos secuencias diferentes: izquierda: spin echo, derecha: spin echo with inversion recovery, cortesía: Matlab.
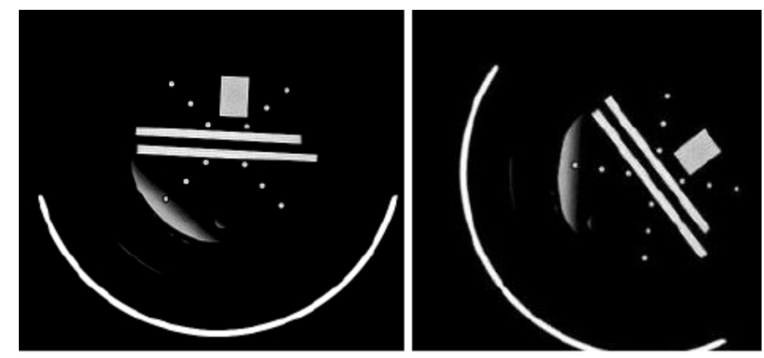

Imagen 4: corte de TAC del phantom de la imagen 1

El set1 se utilizó para calcular la precisión del algoritmo ya que se cuenta con las coordenadas de referencia del phantom. Los set 2 y set 3 se analizaron para determinar las diferencias con un algoritmo similar, el cual forma parte del entorno de Matlab, por el comando IMREGISTER, que realiza un método de registro usando información mutua. Por último, el set4 se usó como control y para pruebas iniciales sobre el algoritmo.

\section{A. Resultados}

En esta sección se analizan los resultados preliminares obtenidos sobre el algoritmo del registro diseñado, el algoritmo se encuentra en fase pruebas y mejoras, con lo que se espera mejorar los resultados en el futuro. Estas pruebas se realizaron bajo transformaciones espaciales de similitud y rígidas.

\section{Interpolador}

Se realizaron pruebas sobre 4 tipos diferentes de interpolador, esta etapa aplicada a la transformación tanto rígida como de similitud afectará el proceso de registro pues inducirá en la imagen artefactos indeseados, que a su vez generarán errores en el algoritmo de comparación, sin embargo, es una etapa fundamental cuando los píxeles luego de la transformación espacial quedan en una posición no rígida. Una comparación entre 4 tipos de interpolador, registrando 15 veces el set1 se puede ver en la tabla 1. Con los otros sets escogidos, por ejemplo: monomodal o algunos sets multimodales, la confiabilidad esta alrededor del $100 \%$, sin importar el método de interpolación y no se apreciaría el cambio en este. Aunque el método de interpolación cúbica tiene un tiempo de procesamiento mayor que el lineal o vecinos más cercanos, hubo menos variación en la alineación final. Se consideró que una desalineación mayor a 4 pixeles resultaría en un fallo en el proceso de registro y así se determinó la confiabilidad. Aunque el cúbico y vecinos más cercanos tuvieron una confiabilidad similar, la alineación obtenida con el cúbico fue más precisa.

\begin{tabular}{|l|c|c|c|c|}
\hline & Cúbico & Lineal & $\begin{array}{c}\text { Vecinos } \\
\text { más } \\
\text { cercanos }\end{array}$ & Lanzcos4 \\
\hline Tiempo(ms) & 7937,2619 & 5560,5003 & 4390,2140 & 15594,6653 \\
\hline Desviación(ms) & 2890,9131 & 1418,3883 & 1321,9696 & 4315,2391 \\
\hline Confiabilidad & $87 \%$ & $73 \%$ & $87 \%$ & $53 \%$ \\
\hline
\end{tabular}

Tabla 1: comparación entre interpoladores.

\section{Optimizador}

Para los procesos de optimización matemática, se probaron 3 tipos de algoritmos basados en computación evolutiva, estos tipos de optimizadores no requieren el uso de derivadas, pero son susceptibles a tener fallos en el proceso de registro, pues en ocasiones reconocen mínimos locales como uno global, esto reducirá la confiabilidad del algoritmo de registro. Entre los tipos de optimizadores probados están: Evolución diferencial (ED), búsqueda Cuco (CS), y optimización por enjambre de partículas (PSO), probado con 3 variantes: PSO con peso inercial adaptativo (PSOAIW), peso inercial caótico (PSOCIW) y decrecimiento lineal (PSOLDIW), ver tabla 2 y 
3. Para cada algoritmo se realizó una prueba de los parámetros libres tales como tamaño del enjambre o la población inicial, número de iteraciones y el mínimo valor de separación entre búsquedas Épsilon, buscando el mejor tiempo de ejecución del algoritmo. Al tener algoritmos diferentes, estos se comportan de manera distintas ante las variaciones de estos parámetros libres.

\begin{tabular}{|c|c|c|c|}
\hline Algoritmo & PSOAIW & PSOCIW & PSOLDIW \\
\hline Tiempo(ms) & 10948,8656 & 11032,3296 & 11197,8152 \\
\hline Desviación(ms) & 82,4222 & 69,5288 & 167,7976 \\
\hline Confiabilidad & $100 \%$ & $100 \%$ & $90 \%$ \\
\hline
\end{tabular}

Tabla 2: algoritmos de optimización basados en PSO

\begin{tabular}{|c|c|c|}
\hline Algoritmo & DE & CS \\
\hline Tiempo(ms) & 36967,4907 & 29881,1259 \\
\hline Desviación(ms) & 157,1377 & 185,6202 \\
\hline Confiabilidad & $100 \%$ & $90 \%$ \\
\hline
\end{tabular}

Tabla 3: Algoritmos de optimización, DE: evolución diferencial y CS: búsqueda Cuco.

PSOAIW presenta las mejores prestaciones tanto en tiempo de procesamiento como en confiabilidad. Los parámetros libres utilizados son: tamaño del enjambre 50, iteraciones 40, y Épsilon de 1.E-7.

\section{B. Precisión}

Para algunas imágenes analizadas, se logra una alineación exacta, como es el caso del set3 o set4, pero como se comentó anteriormente, se realizaron las pruebas de precisión sobre el set1, donde se obtuvieron mayores problemas al registrar, incluso con otros algoritmos como es el caso de Matlab.

En la imagen 5 izquierda, se observa las distancias (D1, D2, D3) y el ángulo, tomadas en la fusión de las imágenes de angiografía y TAC registradas (método 1), se puede observar una desalineación entre estas. En la imagen 5 derecha se toma la imagen registrada de angiografía (método 2), en los puntos 1, 2, 3 y 4, estos se comparan con la imagen de referencia de TAC. Para cada método se tomaron 10 imágenes, producto del algoritmo de registro.
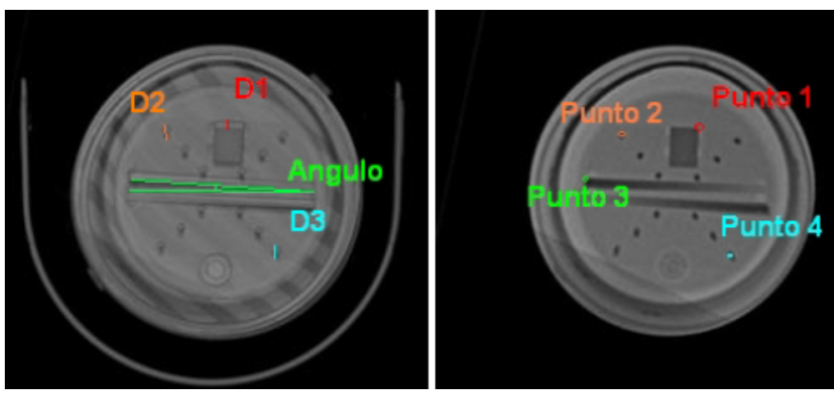

Imagen 5: izquierda: distancias (D1,D2,D3) y el ángulo, imagen fusionada de TAC y angiografía. Derecha: puntos 1,2,3,4 en la imagen de angiografía
En la tabla 4 se pueden apreciar el error en píxeles, tanto promedio, RMS, mínimo y máximo del método 1. Con un error promedio en las distancias medidas en pixeles de $\mathrm{D} 1=3,6, \mathrm{D} 2=6,7$ y $\mathrm{D} 3=5,1$ con un error promedio en el ángulo de 2,48 grados. Al usar el tag DICOM 0028,0030 se pueden convertir los píxeles de la imagen en milímetros, para este caso dicho tag vale 0,8984 , así la distancia promedio es D1=3,2 mm, D2=6,0 $\mathrm{mm}, \mathrm{D} 3=4,5 \mathrm{~mm}$.

\begin{tabular}{|c|c|c|c|c|}
\hline Error & $\begin{array}{c}\text { D1 } \\
\text { (pixeles) }\end{array}$ & $\begin{array}{c}\text { D2 } \\
\text { (pixeles) }\end{array}$ & $\begin{array}{c}\text { D3 } \\
\text { (pixeles) }\end{array}$ & $\begin{array}{c}\text { Angulo } \\
\text { (grados) }\end{array}$ \\
\hline Promedio & 3,65 & 6,70 & 5,45 & 2,63 \\
\hline RMS & 2,59 & 4,87 & 3,87 & 1,91 \\
\hline Mínimo & 3 & 4 & 5 & 1,64 \\
\hline Máximo & 4 & 9 & 6 & 3,24 \\
\hline
\end{tabular}

Tabla 4: distintos tipos de errores, en imágenes fusionadas de TAC, angiografía.

En la tabla 5, para el método 2 se observa el error entre el punto tomado de referencia y el punto en la imagen registrada, se muestra la diferencia en las coordenadas de los puntos 1, 2, 3 y 4 , entre la imagen de referencia TAC (puntos reales) con la imagen de angiografía, ver imagen 5. Se observa poca variación en $\mathrm{X}$ del orden del 0,3\% y una variación mayor en Y del orden del 3,5\%.

\begin{tabular}{|c|c|c|c|c|}
\hline & \multicolumn{2}{|c|}{ Punto 1} & \multicolumn{2}{|c|}{ Punto2 } \\
\hline & $\mathrm{x}$ (pixeles) & $\mathrm{y}$ (pixeles) & $\mathrm{x}$ (pixeles) & $\mathrm{y}$ (pixeles) \\
\hline Promedio & 149,4 & 90,6 & 105,9 & 94,9 \\
\hline Punto real & 150,0 & 87,0 & 106,0 & 91,0 \\
\hline Error & 0,6 & 3,6 & 0,1 & 3,9 \\
\hline $\begin{array}{c}\text { Error } \\
\text { porcentual }\end{array}$ & 0,4 & 4,1 & 0,1 & 4,3 \\
\hline & \multicolumn{2}{|c|}{ Punto3 } & \multicolumn{2}{|c|}{ Punto4 } \\
\hline & $\mathrm{x}$ (pixeles) & $\mathrm{y}$ (pixeles) & $\mathrm{x}$ (pixeles) & $\mathrm{y}$ (pixeles) \\
\hline Promedio & 169,7 & 163,8 & 85,3 & 120,5 \\
\hline Punto real & 170,0 & 161,0 & 85,0 & 116,0 \\
\hline Error & 0,3 & 2,8 & 0,3 & 4,5 \\
\hline $\begin{array}{c}\text { Error } \\
\text { porcentual }\end{array}$ & 0,2 & 1,7 & 0,4 & 3,9 \\
\hline
\end{tabular}

Tabla 5: error entre los puntos 1, 2, 3 y 4, imagen de angiografía comparada con la imagen de referencia (TAC).

\section{Confiabilidad y tiempo de procesamiento}

La confiabilidad y tiempo de procesamiento alcanzado por el algoritmo, varía según cada imagen utilizada en las pruebas, con lo que no se puede sacar un tiempo o confiabilidad promedio, se examinaron diferentes conjuntos de imágenes y se observó su confiabilidad y su tiempo de procesamiento en dos sistemas computaciones diferentes. Para todos se realizó una prueba de 30 registros y se determinó un criterio según las imágenes para determinar si el algoritmo tenia éxito o no registrando.

Con el set1, ver imagen 1, se observa una confiabilidad del orden el $93 \%$ y un tiempo de cómputo de 16161,0765 ms \pm 
948,1103 ms, el registro fue realizado con IMN con interpolador cúbico y algoritmo de optimización PSOAIW con épsilon 0,00017. Los tipos de imágenes multimodales son los más difíciles de registrar con éxito pues las imágenes tienen muchos cambios en su estructura lo que dificulta su registro.

Para el set2, se observó una confiabilidad del $93 \%$ con un tiempo de cómputo promedio de $16834,7450 \mathrm{~ms} \pm 1014,9572$ ms. Para el set3 se observa una confiabilidad del $100 \%$ con un tiempo de cómputo promedio de 22021,2148 ms $\pm 1111,1982$ $\mathrm{ms}$. Ambos se realizaron con transformación rígida e iguales condiciones en el optimizador, sin embargo el set3 tiene un tamaño de $256 \times 256$, mientras que el set 2 tiene un tamaño de $512 \times 512$, pero el set3 de tamaño más pequeño se demoró más que el set 2 de tamaño mayor, se observa que el tiempo de computo no depende del tamaño de las imágenes, sino de las características de estas. Aunque el set 2 y el 3 tienen las mismas imágenes, el set3 es una versión escalada del set2, con lo que se necesitó de algún tipo de interpolación, este proceso pudo inducir la creación de más máximos locales que hacen que el optimizador se demore más tiempo en encontrar la solución óptima.

Para el set4: monomodal, una prueba de confiabilidad se realizó con IM, algoritmo de optimización PSOAIW con Epsilon 0,0017 e interpolador lineal: con una confiabilidad del $100 \%$ y un tiempo de cómputo de 10871,2992 ms $\pm 110,2257$ ms.

Se observó que los resultados acerca de la confiabilidad y tiempo de cómputo del algoritmo dependen de varios factores como:

- El proceso de optimización y la configuración que se aplique, ya que afectan tanto el tiempo de procesamiento como la confiabilidad, pocas iteraciones pueden reducir el tiempo de cómputo, pero provocará una mayor cantidad de fallas; una población inicial muy grande puede mejorar la confiabilidad, pero aumenta el tiempo de procesamiento; una épsilon muy pequeña podrá mejorar el registro, pero aumentando considerablemente el tiempo de procesamiento, entre otros.

- El tipo de transformación, ya sea de similitud o rígida, afectan el tiempo de procesamiento y los parámetros en el optimizador que deben ser diferentes ya sea para una o la otra. La transformación de similitud toma más tiempo en ejecutarse, pero entrega mejor resultados en algunos casos. La transformación rígida se ejecutará más rápido, pero en algunos casos no muestra buenos resultados, sin embargo, puede ser suficiente para algunas imágenes.
- El interpolador usado afecta la confiabilidad del algoritmo. Se recomienda usar interpolador cúbico para imágenes multimodales, pues reduce artefactos en las imágenes que pueden generarse con otros interpoladores.

Al implementar las técnicas de registro en el sistema de cómputo para Neuronavegación se observan variaciones del tiempo de cómputo para los sets 1,2 y 3 . El equipo donde está instalado el sistema es un Intel Core Vpro I7-4940MX $3.10 \mathrm{GHz}$ con 4 procesadores físicos y 8 hilos, con $32 \mathrm{~Gb}$ de RAM a $1600 \mathrm{MHz}$. Para el set 1 se obtuvo un promedio de $9919,7228 \pm 1282,2815 \mathrm{~ms}$, para el set $27596,6261 \pm$ $332,9386 \mathrm{~ms}$ y el $38577,8095 \pm 860,8870 \mathrm{~ms}$. Comparado con los tiempos obtenidos en las pruebas anteriores, para el set 1 se redujo en $38 \%$, para el 2 se redujo un $54 \%$ y para el 3 se redujo $61 \%$. Se debe recalcar que no se están utilizando todos los núcleos del procesador.

\section{Robustez y cambios entre la IM y IMN}

Se analizó el comportamiento del algoritmo frente a ruido del tipo gaussiano en la imagen y como filtros reductores de ruido afectaran a este, entre los filtros probados están: filtro gaussiano, mediano y Weiner. También se analizó el algoritmo bajo condiciones correcciones gamma y bajo ecualizaciones del histograma usando una ecualización tradicional (HE) y una adaptativa con limitador de contraste (CLAHE). La corrección gamma, es una operación no lineal que aumenta o disminuye el brillo de la imagen, las operaciones de ecualización buscan tener un histograma más uniforme con lo que aumenta el contraste de la imagen. Dichas operaciones son comunes en el procesamiento digital de imágenes por lo tanto se decidió estudiar sus efectos. Para estas pruebas, se usaron el set3 (multimodal) y 4 (monomodal).

En la tabla 6, se observa como varia el error porcentual de la información mutua (IM) y su versión normalizada (IMN) ante ruido gaussiano, con varianzas diferentes $\mathrm{V}$, con una imagen monomodal. Se aprecia que la IMN tiene un error reducido del orden del $30 \%$ que la IM la cual tiene un error mayor.

\begin{tabular}{|c|c|c|c|c|}
\hline Monomodal & IM & $\begin{array}{c}\text { Error } \\
(\%)\end{array}$ & IMN & $\begin{array}{c}\text { Error } \\
(\%)\end{array}$ \\
\hline Sin ruido & 2,9994 & $0.00 \%$ & 1,5379 & $0.00 \%$ \\
\hline $\mathrm{V}=0,01$ & 0,6560 & $78,13 \%$ & 1,6314 & $30,96 \%$ \\
\hline $\mathrm{V}=0,02$ & 0,4695 & $84,35 \%$ & 1,0414 & $32,17 \%$ \\
\hline $\mathrm{V}=0,03$ & 0,9834 & $87,22 \%$ & 1,0347 & $32,69 \%$ \\
\hline
\end{tabular}

Tabla 6: Comportamiento de la IM y la IMN frente al ruido gaussiano con diferentes variancias, en una imagen monomodal

En la tabla 7, se observa un comportamiento similar al de la tabla 6, pero con una imagen multimodal. La IMN posee un mejor rendimiento que la no normalizada. 


\begin{tabular}{|c|c|c|c|c|}
\hline Multimodal & IM & $\begin{array}{c}\text { Error } \\
(\%)\end{array}$ & IMN & $\begin{array}{c}\text { Error } \\
(\%)\end{array}$ \\
\hline Sin ruido & 1,2783 & $0.00 \%$ & 1,1857 & $0.00 \%$ \\
\hline $\mathrm{V}=0,01$ & 0,6448 & $49,56 \%$ & 1,0675 & $9,97 \%$ \\
\hline $\mathrm{V}=0,02$ & 0,4879 & $61,83 \%$ & 1,0494 & $11,50 \%$ \\
\hline
\end{tabular}

Tabla 7: Comportamiento de la IM y la IMN frente al ruido gaussiano con diferentes variancias, en una imagen multimodal

En la tabla 8 se observa el comportamiento de la IM y la IMN frente a algunos filtros reductores de ruido: mediano y Weiner con ventanas $3 \times 3$ y $5 \times 5$, se probó con una imagen multimodal. La IMN tiene una menor variación, sin embargo, los filtros aumentaron la información mutua entre las imágenes, esto mejora el comportamiento del algoritmo.

\begin{tabular}{|c|c|c|c|c|}
\hline & IM & Error (\%) & IMN & Error (\%) \\
\hline Sin filtro & 1,1857 & $0,00 \%$ & 1,1833 & $0,00 \%$ \\
\hline Mediano 3x3 & 1,3449 & $13,43 \%$ & 1,1989 & $1,32 \%$ \\
\hline Mediano 5x5 & 1,1989 & $1,11 \%$ & 1,2101 & $2,26 \%$ \\
\hline Weiner 3x3 & 1,3935 & $17,53 \%$ & 1,1971 & $1,17 \%$ \\
\hline Weiner 5x5 & 1,3538 & $14,18 \%$ & 1,2067 & $1,98 \%$ \\
\hline
\end{tabular}

Tabla 8: Comportamiento de la IM y la IMN frente a filtros reductores de ruido.

Variación de la IM y la IMN con respecto al factor gamma: en la tabla 9, se observa como varia la IMN y la IM, ante una corrección gamma, una corrección gamma es un proceso que cambia el valor de la intensidad del pixel con una transformación no lineal como se ve en [12], esta operación altera el brillo de la imagen. Se observa que la IMN tiene una mejor respuesta ante esta alteración, pero tanto la IM como la IMN poseen poca variación con un factor Gamma de 0,6 en adelante para la IM y 0,4 para la IMN. Se determina también si el proceso de registro tiene éxito o no.

\begin{tabular}{|c|c|c|c|c|c|c|}
\hline Gamma & IMN & Error(\%) & Éxito & IM & Error(\%) & Éxito \\
\hline 1 & 1,1857 & $0,00 \%$ & si & 1,2783 & $0,00 \%$ & si \\
\hline 0,2 & 1,0192 & $14,04 \%$ & no & 0,0869 & $93,20 \%$ & no \\
\hline 0,4 & 1,1568 & $2,44 \%$ & si & 0,8755 & $31,51 \%$ & si \\
\hline 0,6 & 1,1708 & $1,26 \%$ & si & 1,0549 & $17,48 \%$ & si \\
\hline 0,8 & 1,1896 & $0,33 \%$ & si & 1,2479 & $2,38 \%$ & si \\
\hline 1,5 & 1,1806 & $0,43 \%$ & si & 1,2956 & $1,35 \%$ & si \\
\hline 2 & 1,1792 & $0,55 \%$ & si & 1,3002 & $1,71 \%$ & si \\
\hline 2,5 & 1,1788 & $0,58 \%$ & si & 1,3003 & $1,72 \%$ & si \\
\hline
\end{tabular}

Tabla 9: Comportamiento de la IMN y la IM frente a correcciones Gamma.

Variación de la IM y la IMN con respecto a la ecualización del histograma: en esta prueba se observó la variación de la IM y la IMN cuando se cambia el contraste de la imagen. El objetivo de la ecualización es hacer más uniforme el histograma, mejorando el contraste de la imagen. Entre los métodos usados tenemos ecualización del histograma tradicional (HE, Histogram ecualization) [13] y CLAHE
(Contrast Limited Adaptive Histogram Equalization), [14]. En la tabla 10 se observa la variación de los métodos, se mide la entropía de las imágenes 1 o 2 para notar los cambios en la información mutua. La entropía de la imagen 1 sin ecualizar es de 4,2666 y de la imagen 2 es de 3,8958, la información mutua de las imágenes sin ecualizar es de 1,2783, la información mutua normalizada es de 1,1857 . IMN posee menos variación, sin embargo, hay poco cambio ante este tipo de procedimientos.

\begin{tabular}{|c|c|c|c|c|c|c|}
\hline Método & $\begin{array}{c}\text { Entropía } \\
1\end{array}$ & $\begin{array}{c}\text { Entropía } \\
2\end{array}$ & IM & $\begin{array}{c}\text { Error } \\
(\%)\end{array}$ & IMN & $\begin{array}{c}\text { Error } \\
(\%)\end{array}$ \\
\hline HE & 4,2666 & 3,8958 & 1,2046 & 5,77 & 1,1880 & 0,19 \\
\hline CLAHE & 4,2666 & 3,8958 & 1,3017 & 1,83 & 1,1627 & 1,94 \\
\hline
\end{tabular}

Tabla 10: Comportamiento de la IM y la IMN frente a métodos de ecualización del contraste.

Se demuestra por tanto que la IMN, es más robusta que la versión no normalizada, esto es congruente con las investigaciones realizadas sobre el tema, como se aprecia en [1]. Estas pruebas también demostraron que la IMN es una mejor opción para el algoritmo diseñado. Conforme a la literatura respecto al tema, la IM y la IMN tiene una susceptibilidad al ruido, reducido para el caso de la IMN, pero poca variación ante cambios en el contraste y el brillo. Una etapa de reducción de ruido como se aprecia en la tabla 8 , mejora la información mutua y por ende mejora el proceso de registro.

\section{CONCLUSIONES}

La información mutua demostró ser una herramienta capaz de registrar imágenes multimodales y monomodales, sin embargo, algunos artefactos en las imágenes a registrar pueden ocasionar errores en los procesos de registro. Sobre todo, en los compuestos por imágenes multimodales, donde se logra una confiabilidad del entre el $87 \%$ al $93 \%$ con las imágenes probadas. Pero en muchos otros casos se logró una precisión y confiabilidad cercanas al 100\%.

La mejor opción para la etapa de comparación fue usar información mutua normalizada, pues se observó un mejor desempeño con respecto a la información mutua estándar, como en el efecto del ruido gaussiano o variación del brillo o el contraste. También se apreció una reducción en el tiempo de procesamiento posiblemente porque se eliminan varios máximos locales en la función, esto coincide con lo que se reporta en la literatura investigada.

En la etapa de transformación espacial el mejor método de interpolación fue el interpolador cúbico, aunque requiere más tiempo de procesamiento, genera menos artefactos indeseables en la imagen que pueden afectar el proceso de registro y generar fallas en este. Sin embargo, en varias situaciones consideradas el uso de otros interpoladores resultó óptima y con un menor tiempo de procesamiento. 
El mejor algoritmo encontrado dentro de los analizados fue el PSOAIW, dicho algoritmo fue capaz de buscar la mejor solución en varios sets analizados con una tasa de acierto cercano al $100 \%$, sin embargo, en algunas imágenes, tuvo una tasa de éxito reducida de hasta $87 \%$, esto se atribuye a la cantidad de máximos locales que se generan en el espacio de búsqueda, debido a condiciones propias de las imágenes o artefactos inducidos en las etapas de interpolación en las transformaciones espaciales.

\section{AGRADECIMIENTOS}

La investigación relacionada a este documento fue ejecutada gracias al Instituto de Epilepsia y Parkinson del Eje Cafetero NEUROCENTRO S.A y a Colciencias, a través del "Programa nacional de ciencia y tecnología en salud", con el proyecto titulado: "Desarrollo de pruebas de concepto de un dispositivo médico de navegación cerebral tridimensional: aplicación a procesos neuroquirúrgicos", código: 499170049019.

\section{REFERENCIAS}

[1]. A. Ardeshir Goshtasby. Image Registration Principles, Tools and Methods. Advances in Computer Vision and Pattern Recognition. Springer, 2012

[2]. Stefan Klein y Marius Staring. Elastix the manual. University Medical Center Utrecht, 2012. [Online]. Available: http://elastix.isi.uu.nl/download/elastix_manual_v4.8 .pdf

[3]. G.P. Penney, J.Weese, J.A. Little, P. Desmedt, D.L.G. Hill, y D.J. Hawkes. A comparison of similarity measures for use in 2-D-3-D medical image registration. Medical Imaging, IEEE Transactions on, pp. 586-595, 1998.

[4]. Derek L.G. Hill y David J. Hawkes. Handbook ofMedical Image Processing and Analysis Chapter 37 - Across-Modality Registration Using IntensityBased Cost Functions. Academic Press, Burlington, second edition edition, 2009.

[5]. Roger P. Woods. Handbook of Medical Image Processing and Analysis Chapters 32-33. Academic Press, Burlington, second edition, 2009.
[6]. Xiu Ying Wang, Stefan Eberl, Michael Fulham, Seu Som, y David Dagan Feng. 8 - Data Registration and Fusion. Biomedical Engineering. Academic Press, Burlington, 2008.

[7]. C. Studholme, D. L. G. Hill, and D. J. Hawkes, "An overlap invariant entropy measure of $3 \mathrm{D}$ medical image alignment", Pattern Recognit., vol. 32, no. 1, pp. 71-86, 1999

[8]. Sima Noghanian, Abas Sabouni, Travis Desell, y AliAshtari. Microwave Tomography: Global Optimization, Parallelization and Performance Evaluation. Springer Publishing Company, Incorporated, 2014.

[9]. Xin-She Yang y S. Deb. Cuckoo search via levy fights. In Nature Biologically Inspired Computing, 2009. NaBIC 2009. World Congress on, pages 210214, Dec 2009.

[10]. Lin Lu, Qi Luo, Jun yong Liu, y Chuan Long. An improved particle swarm optimization algorithm. In Granular Computing, 2008. IEEE International Conference on, pages 486-490, Aug 2008.

[11]. A. Ardeshir Goshtasby. 2D and 3-D Image Registration: For Medical, Remote Sensing, and Industrial Applications. Wiley-Interscience, 2005.

[12]. Doustar, M.F.; Hassanpour, H., "A locallyadaptive approach for image gamma correction," in Information Sciences Signal Processing and their Applications (ISSPA), 2010 10th International Conference on, pp.73-76, 2010

[13]. Soong-Der Chen, Abd. Rahman Ramli, Preserving brightness in histogram equalization based contrast enhancement techniques, Digital Signal Processing, Volume 14, Issue 5, Pages 413$428,2004$.

[14]. Zuiderveld, K.: 'Contrast limited adaptive histogram equalization', inHeckbert, P.S. (Eds.): 'Chapter VIII, graphics gems IV', AcademicPress, Cambridge, MA, pp. 474-485, 1994. 